\title{
Bronchiolitis obliterans syndrome in lung transplant recipients is associated with increased neutrophil activity and decreased anti- oxidant status in the lung
}

\author{
G.C. Riise*, A. Williams+, C. Kjellström**, H. Schersten***, B.A. Andersson*, F.J. Kelly+
}

\begin{abstract}
Bronchiolitis obliterans syndrome in lung transplant recipients is associated with increased neutrophil activity and decreased antioxidant status in the lung. G.C. Riise, A. Williams, C. Kjellström, H. Schersten, B.A. Andersson, F.J. Kelly. OERS Journals Ltd 1998.

ABSTRACT: Long-term survival of lung transplant recipients is limited by the advent of obliterative bronchiolitis and irreversible airways obstruction, e.g. bronchiolitis obliterans syndrome (BOS). This study investigated whether inflammatory cells and their activation markers were increased in bronchoalveolar lavage (BAL) and transbronchial biopsies (TBB) from patients with BOS. Levels of antioxidants in BAL fluid were also assessed.

BAL fluid and TBB from six single-lung, two bilateral-lung, and five heart-lung transplanted patients with diagnosis of $\mathrm{BOS}$ were compared with 13 transplant recipients without BOS. BAL fluid levels of myeloperoxidase (MPO), eosinophil cationic protein (ECP) and interleukin (IL)-8 were used as markers for the activation and attraction of neutrophils and eosinophils, respectively. Immunohistochemical staining of TBB with monoclonal antibodies to MPO and ECP (EG2) was performed.

Significantly increased BAL percentages of neutrophils and levels of MPO were found in patients with BOS. The findings correlated well with the degree of monoclonal staining for MPO in TBB. BAL levels of ECP and IL-8 were significantly increased in BOS patients. BAL concentrations of the water-soluble antioxidants ascorbate, urate and glutathione were generally lower in BOS patients.

The results indicate that neutrophil infiltration and activation, as well as oxidative stress, may play a role in the development and/or progression of bronchiolitis obliterans syndrome. Markers for neutrophil activation could have a potential role in monitoring disease activity in patients with this syndrome.

Eur Respir J 1998; 12: 82-88.
\end{abstract}

Depts of *Pulmonary Medicine, **Pathology and $* * *$ Cardiothoracic Surgery, Sahlgrenska University Hospital, Göteborg, Sweden. ${ }^{+}$Cardiovascular Research, The Rayne Institute, St Thomas' Hospital, London, UK. ‡Dept of Clinical Immunology, University of Göteborg, Göteborg, Sweden.

Correspondence: G.C. Riise

Dept of Pulmonary Medicine

Sahlgrenska University Hospital

S-413 45 Göteborg

Sweden

Fax: 4631824904

Keywords: Antioxidants

bronchiolitis obliterans syndrome

interleukin-8

lung transplantation

myeloperoxidase

neutrophils

Received: October 201997

Accepted after revision April 81998

Supported by the Swedish Heart and Lung Foundation, the Medical Society of Göteborg and the Swedish Medical Society.

In lung transplant recipients, long-term survival is limited by the advent of chronic rejection, a severe complication in the form of obliterative bronchiolitis (OB). It is generally diagnosed in the second postoperative year and affects at least $30-40 \%$ of lung transplanted patients [1].

Clinically, rejection of the lung allograft is diagnosed by a combination of histopathological assessment of transbronchial biopsies (TBB) and cytological assessment of bronchoalveolar lavage (BAL) samples. Acute rejection is characterized by a perivascular lymphocytic infiltration in the parenchyma with or without concomitant lymphocytic bronchitis/bronchiolitis [2]. Chronic rejection is diagnosed as a therapy-resistant progressive loss of lung function with the histopathological finding of OB in TBB [3]. However, since changes typical of $\mathrm{OB}$ are not often present in the usually minute TBB specimens [4], the clinical definition of bronchiolitis obliterans syndrome (BOS) has been based on both spirometric and biopsy findings [3]. Less invasive methods than TBB as well as more sensitive predictors for the onset of BOS are needed for the diagnosis of chronic rejection in the transplanted lung.

In a recent investigation of specific markers reflecting the activation of inflammatory cells, no evidence was found that BAL fluid levels of eosinophil cationic protein (ECP) from activated eosinophils or myeloperoxidase (MPO) from activated neutrophils were associated with acute lung rejection. Interleukin (IL)-8, with a known chemotactic effect on neutrophil granulocytes, was also unaffected [5]. Likewise, although antioxidant status is known to be compromised in lung transplant recipients [6], no further deterioration of these defences is seen during periods of acute rejection [7]. In chronic rejection, however, a recent study reported both increased BAL levels of IL-8, BAL neutrophilia and immunolocalization of IL-8 in the peribronchial regions of patients with $\mathrm{OB}$ [8]. It is hypothesized that such increase in the numbers of activated neutrophils in the lung could give rise to changes in BAL fluid antioxidant defences due to an increased release of reactive oxygen species.

The present study was designed to investigate further the pattern of inflammatory cells as well as their corresponding activation markers in both BAL fluid and TBB from lung transplant recipients with BOS. Levels of antioxidants in BAL fluid were assessed. The aim was to see whether any of these variables were also associated with the presence of BOS, compared with matched control subjects without BOS. 


\section{Methods}

\section{Design}

During a period from February 1996 to February 1997 (12 months), BAL fluid was collected from all diagnostic and protocol bronchoscopies performed on the 80 patients examined in the lung transplant program of Sahlgrenska University Hospital (Göteborg, Sweden). From this material, BAL samples from 13 subjects with a diagnosis of BOS were obtained. The control group comprised 13 matching lung transplant recipients without BOS, but otherwise similar with respect to immunosuppressive therapy, age, sex, type of lung transplantation, absence of acute rejection, and time postsurgery for control bronchoscopy. Except for one subject, the presence of infection was controlled for. The study design was approved by the ethical committee of the University of Göteborg.

\section{Subjects}

Thirteen single-lung $(n=6)$, bilateral-lung $(n=2)$, or heart-lung $(n=5)$ transplant patients with a diagnosis of BOS were identified and 13 single-lung $(n=6)$, bilaterallung $(n=3)$, or heart-lung $(n=4)$ transplant patients were used as controls. Organ donors and recipients were matched for cytomegalovirus (CMV) serological status and all organs had been harvested in a similar fashion. Surgical procedures and immunosuppression therapy were performed as described previously [9]. For detailed patient characterization, see table 1 .

\section{Postoperative follow-up}

The postoperative follow-up design of the lung transplant programme of Sahlgrenska University Hospital has been described in detail previously [5]. In short, protocol bronchoscopies with $\mathrm{TBB}$ and BAL were performed at regular intervals post-transplant, and whenever indicated by a worsening of the clinical condition of the patient. Fibreoptic bronchoscopy was performed transorally with local anaesthesia and intravenous propofol sedation. Supplemental $100 \%$ oxygen was delivered nasally and transcutaneous blood oxygen saturation continually monitored with an Ohmeda pulse oximeter (Ohmeda, Louisville, KY, USA).

Lung biopsy evaluation of acute rejections followed the recommendations of the lung rejection study group of the International Society for Heart and Lung Transplantation (ISHLT) [2], and chronic rejection with OB according to the grading system established by COOPER et al. [3].

BAL analysis included direct microscopy for CMV inclusion bodies, Pneumocystis carinii (PCP), fungi and mycobacteria, and cultures for bacteria including Legionella and mycobacteria, fungi and viruses. Immunocytochemistry techniques were used for the identification of PCP, CMV and Legionella pneumophilia in BAL and/or TBB samples. In addition, the presence of CMV and respiratory syncytial virus (RSV) genome were investigated by polymerase chain reaction (PCR) amplification. Histological diagnostic criteria for CMV pneumonitis were viral inclusion bodies ("owl's eye"), positive immune reaction or both in TBB or BAL, together with a parenchymal, diffuse or perivascular, inflammation. This definition of an active CMV pneumonitis fulfils the criteria for CMV disease of the Fourth International Cytomegalovirus Workshop [10]. Bacterial infection was diagnosed by a significant bacterial growth in the BAL sample $\left(>10^{5}\right.$ colony forming units (cfu) $\left.\cdot \mathrm{mL}^{-1}\right)$, and diagnosis of PCP was based on demonstration of the organism by silver-staining and immunohistochemistry in TBB and BAL samples.

\section{Collection of samples}

All bronchoscopies were performed in the morning, before 10:30 h. BAL was obtained by infusion of $2 \times 3 \times 20$ $\mathrm{mL}$ warmed sterile pyrogen-free phosphate-buffered saline (PBS) solution into a segmental middle lobe or lingula bronchus with the bronchoscope in a wedged position. The

Table 1. - Clinical characteristics of lung transplant recipients with and without bronchiolitis obliterans syndrome (BOS)

\begin{tabular}{|c|c|c|c|c|c|c|c|c|c|c|c|c|c|}
\hline \multicolumn{7}{|c|}{ With BOS } & \multicolumn{7}{|c|}{ Without BOS } \\
\hline $\begin{array}{l}\text { Preop } \\
\text { diagnosis }\end{array}$ & $\mathrm{Tx}$ & $\begin{array}{c}\text { Age } \\
\text { at Tx } \\
\text { yrs }\end{array}$ & Sex & $\begin{array}{l}\text { Months } \\
\text { post-Tx }\end{array}$ & $\begin{array}{c}\text { Degree } \\
\text { of } \\
\text { BOS }\end{array}$ & Infect & $\begin{array}{l}\text { Preop } \\
\text { diagnosis }\end{array}$ & $\mathrm{Tx}$ & $\begin{array}{c}\text { Age } \\
\text { at Tx } \\
\text { yrs }\end{array}$ & Sex & $\begin{array}{l}\text { Months } \\
\text { post-Tx }\end{array}$ & $\begin{array}{c}\text { Degree } \\
\text { of } \\
\text { BOS }\end{array}$ & Infect \\
\hline$\overline{\alpha_{1}-\mathrm{AT}}$ & SL & 53 & $\mathrm{~F}$ & 24 & 3B & No & Emphysema & SL & 56 & $\mathrm{~F}$ & 24 & 0 & No \\
\hline Pulmonary fibrosis & SL & 51 & $\mathrm{~F}$ & 21 & $2 \mathrm{~B}$ & No & $\alpha_{1}-\mathrm{AT}$ & SL & 51 & $\mathrm{~F}$ & 24 & 0 & No \\
\hline Eisenmenger & HL & 16 & M & 43 & 3B & No & Eisenmenger & HL & 19 & M & 36 & 0 & No \\
\hline PPH & HL & 44 & $\mathrm{~F}$ & 18 & $2 \mathrm{~A}$ & No & Eisenmenger & HL & 40 & $\mathrm{~F}$ & 19 & 0 & No \\
\hline Emphysema & SL & 46 & $\mathrm{~F}$ & 18 & 2B & No & Emphysema & SL & 45 & $\mathrm{~F}$ & 18 & 0 & No \\
\hline Emphysema & SL & 54 & $\mathrm{~F}$ & 26 & 2B & No & Emphysema & SL & 54 & $\mathrm{~F}$ & 24 & 0 & No \\
\hline Pulmonary fibrosis & SL & 44 & M & 36 & $2 \mathrm{~A}$ & No & Pulmonary fibrosis & SL & 50 & M & 48 & 0 & No \\
\hline$\alpha_{1}-\mathrm{AT}$ & BL & 51 & $\mathrm{~F}$ & 10 & 3B & No & Emph & BL & 51 & $\mathrm{~F}$ & 9 & 0 & No \\
\hline Emphysema & SL & 52 & $\mathrm{~F}$ & 48 & $2 \mathrm{~A}$ & No & Emphysema & SL & 54 & $\mathrm{~F}$ & 48 & 0 & No \\
\hline Cystic fibrosis & HL & 21 & M & 58 & 3B & No & Cystic fibrosis & $\mathrm{BL}$ & 25 & $\mathrm{~F}$ & 48 & 0 & No \\
\hline $\mathrm{PPH}$ & HL & 24 & $\mathrm{~F}$ & 140 & $2 \mathrm{~B}$ & Yes* & $\mathrm{PPH}$ & HL & 29 & M & 108 & 0 & No \\
\hline Eisenmenger & HL & 44 & M & 24 & $2 \mathrm{~A}$ & No & $\alpha_{1}-\mathrm{AT}$ & $\mathrm{BL}$ & 37 & M & 24 & 0 & No \\
\hline Pulmonary fibrosis & $\mathrm{BL}$ & 55 & $\mathrm{~F}$ & 118 & $2 \mathrm{~B}$ & No & Eisenmenger & $\mathrm{HL}$ & 27 & $\mathrm{~F}$ & 81 & 0 & No \\
\hline
\end{tabular}

Patients (9 females (F), 4 males (M)) with BOS (n=13) had median age 46 yrs, median post-transplantation (Tx) 26 months and 1 out of 13 had infection (Infect). Patients (9F, 4M) without BOS $(n=13)$ had median age 45 yrs, median post-Tx 24 months and none had infection. Preop: preoperative; $\alpha_{1}$-AT: antitrypsin deficiency; PPH: primary pulmonary hypertension; SL: single-lung; HL: heart-lung; BL: bilateral-lung; *: Haemophilus influenzae. 
fluid was aspirated after each $60 \mathrm{~mL}$ infusion, collected in a sterile siliconized container and immediately transported on ice to the laboratory. After filtering, cellular components were sedimented by centrifugation at $4^{\circ} \mathrm{C}$ and 200 $\times \mathrm{g}$ for $10 \mathrm{~min}$, and the supernatant removed. After an additional 10 -min centrifugation at $10,000 \times \mathrm{g}$, the supernatant was frozen and stored at $-70^{\circ} \mathrm{C}$.

Cytocentrifuge slides (Shandon Southern Products, Runcorn, UK) were made from $100 \mu \mathrm{L}$ aliquots of the resuspended cell pellet. Slides were fixed in $96 \%$ alcohol and stained with May-Grünwald-Giemsa for later identification of cell types on a morphological basis. Percentages of eosinophil granulocytes, neutrophil granulocytes, macrophages and lymphocytes were calculated by counting 200 cells using a standard light microscope. All samples were analysed in a blinded manner.

TBB were always taken after BAL. At least five macroscopically adequate biopsy specimens were taken under fluoroscopic guidance from the lower and middle lobes of one lung using alligator forceps, immediately placed in $10 \%$ buffered formalin and sent for histopathological analysis.

\section{Immunohistochemical analysis of transbronchial lung biopsies}

All biopsies fulfilled the criteria of evaluation established by the ISHLT [2]. The paraffin-embedded TBB samples used for routine histopathology were sectioned, deparaffinized and hydrated. A monoclonal antibody to myeloperoxidase (MPO, no. A398; Dakopattts, Stockholm, Sweden) was used at a dilution of 1:2000 and a monoclonal antibody to ECP (EG2, no. 10-9196-01; Kabi Pharmacia Diagnostics, Uppsala, Sweden) at a dilution of 1: 100. The standard avidin-biotin method of immuno-staining [11] was performed on one section of each biopsy and appropriate positive and negative controls were used in every staining run. The immunopositivity of the entire biopsy section was assessed and the bronchial, alveolar and/or interstitial distribution of positive staining evaluated semiquantitatively by one observer in a blinded manner on two separate occasions (weighted Kappa value 0.85 (95\% confidence interval $0.76-0.95)$ ). Results were expressed on a scale between 0 and 2, e.g. degree 0 for no positivity, 1 for scattered positive cells (fig. 1) and 2 when the biopsies contained groups of immunopositive cells (fig. 2).

\section{Analysis of inflammatory markers in bronchoalveolar lavage fluid}

BAL fluid analysis of ECP [12] (Pharmacia Diagnostics, Uppsala, Sweden), MPO [13] (Pharmacia) and albumin (Pharmacia) was performed using radioimmunoassays according to the instructions of the manufacturer. The intercoefficients and intracoefficients of variation of the assays have been accounted for in the instructions issued by the manufacturer. IL-8 (ImmunoKontact, Frankfurt am Main, Germany) was measured by enzyme-linked immunosorbent assay (ELISA) using the following protocol: capture antibody, $2 \mu \mathrm{g} \cdot \mathrm{mL}^{-1}$ in 0.1 $\mathrm{M} \mathrm{NaHCO}$ buffer, $\mathrm{pH} 8.2$, was coated into the wells of a 96-well plate (Maxisorp; Nunc, Roskilde, Denmark) overnight at $4^{\circ} \mathrm{C}$. After two washes with PBS-Tween $0.05 \%$, the plate was block-ed with $3 \%$ bovine serum albumin (BSA) in PBS for $2 \mathrm{~h}$ at room temperature. The samples were added and the plate was incubated overnight at $4^{\circ} \mathrm{C}$. After four washes with PBS-Tween, the biotinylated antibody, $1 \mu \mathrm{g} \cdot \mathrm{mL}^{-1}$ in PBS-3\% BSA, was added and the plate was incubated for $1 \mathrm{~h}$ at room temperature. After six washes extravidin was added and incubated. After eight washes, substrate was added and the absorbance was measured at $405 \mathrm{~nm}$ in a

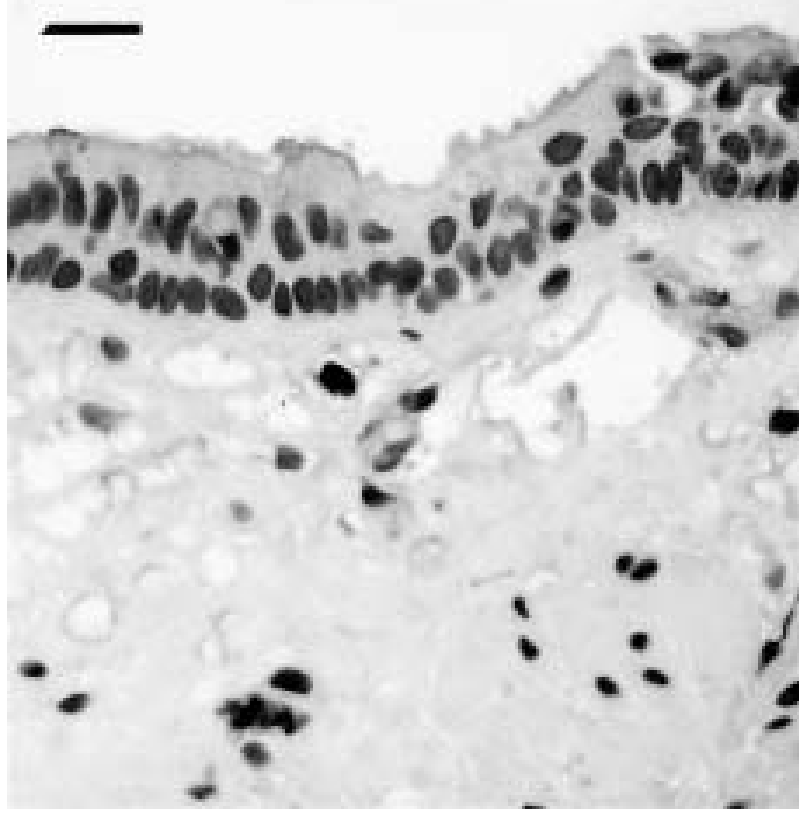

Fig. 1. - Transbronchial biopsy from subject with bronchiolitis obliterans syndrome with scattered myeloperoxidase-positive cells in the bronchiolar wall (degree 1 positivity). (Internal scale bar=100 $\mu \mathrm{m}$ ).

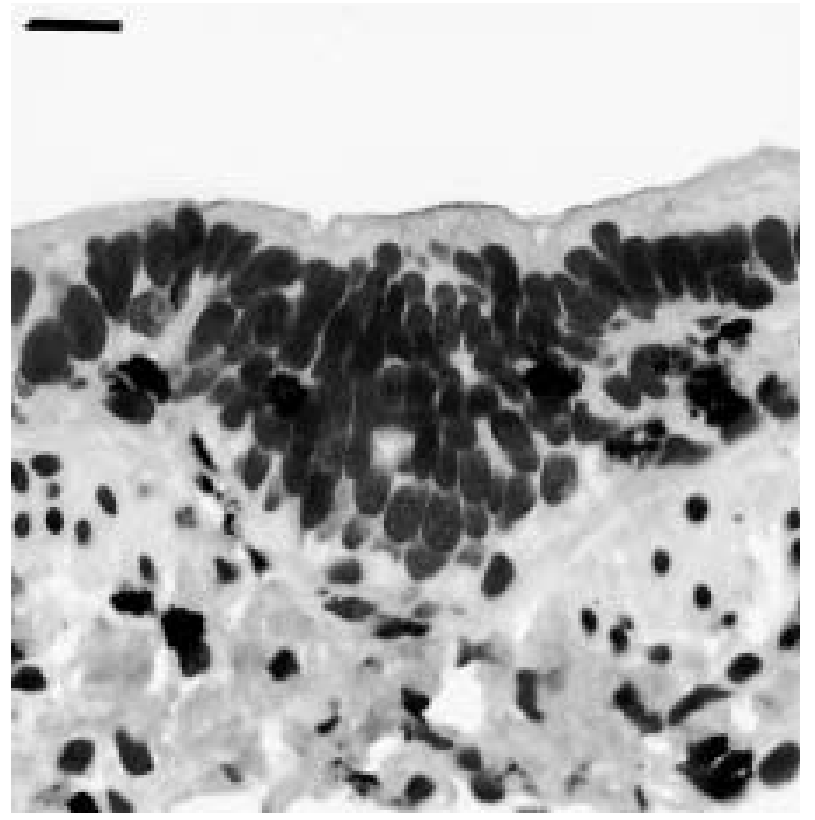

Fig. 2. - Transbronchial biopsy from subject with bronchiolitis obliterans syndrome with clusters of myeloperoxidase-positive cells in the bronchiolar wall (degree 2 positivity). (Internal scale bar $=50 \mu \mathrm{m}$ ). 
spectrophotometer (Titertek Multiscan, Flow Laboratories, Maclean, USA). The level of sensitivity for the ELISA was $15.6 \mathrm{pg} \cdot \mathrm{mL}^{-1}$.

\section{Antioxidant determinations}

Ascorbic acid and uric acid concentrations were determined using a high-performance liquid chromatography (HPLC) method based on that of IRIYAMA et al. [14] and as reported recently [15]. A microplate reader adaptation [15] of the enzyme recycling method described by TIETZE [16] was used for the determination of reduced (GSH) and oxidized (GSSG) glutathione.

\section{Statistical evaluation}

The individuals performing each assay were blinded as to the classification of samples. Results are expressed as medians and ranges. Analysis of differences between the patient groups was performed with the Mann-Whitney U-test and for correlations the Spearman Rank correlation test was used. Statistically significance was noted at $\mathrm{p}<$ 0.05 .

\section{Results}

No statistically significant differences were found between the study groups for age, female/male ratio or the time after transplantation when the samples were obtained. Blood cyclosporine levels (median $215 \mathrm{ng} \cdot \mathrm{mL}^{-1}$, range 170-270 in BOS patients; median $225 \mathrm{ng} \cdot \mathrm{mL}^{-1}$, range 150-300 in controls) and prednisone medication (median $7.5 \mathrm{mg}$, range 5-15 mg in BOS; median $5 \mathrm{mg}$, range 5-10 $\mathrm{mg}$ in controls) were comparable. None of the subjects in the study had a diagnosis of acute rejection. No subjects had any diagnosis of infection, except for one patient with BOS grade $3 \mathrm{~B}$ with a diagnosis of Haemophilus influenzae bacterial infection.

A perfect match between groups was not achieved since two pairs of patients were of a different sex and in three pairs of patients, heart-lung transplant subjects were compared to bilateral-lung transplant recipients. In seven pairs, identical preoperative diagnoses were compared. In two, $\alpha_{1}$-antitrypsin deficiency was compared with emphysematous disease, and in one pair primary pulmonary hypertension was compared with Eisenmenger syndrome. In three subject pairs, no matching for preoperative diagnosis could be achieved (table 1).

Nine patients had both clinical and histopathological criteria for BOS (five grade $2 \mathrm{~B}$ and four grade $3 \mathrm{~B}$ ), whereas four patients fulfilled the clinical criteria for BOS grade $2 \mathrm{~A}$ (table 1).

\section{Polymorphonuclear granulocytes in TBB}

The semiquantitative assessment of immunohistochemical positivity for MPO and presence of activated neutrophils in the bronchial wall was significantly higher in patients with BOS (median 2, range 1-2) compared with controls (median 0 , range $0-2, \mathrm{p}<0.01$ ) (figs. 1 and 2 ). The degree of MPO positivity in the alveolar spaces or the interstitium did not differ significantly between the groups.

The degree of MPO positivity in the bronchial wall correlated significantly both with percentages of neutrophils in BAL $(\mathrm{p}<0.01, \mathrm{r}=0.6)$ and with levels of MPO in BAL $(\mathrm{p}<0.01, \mathrm{r}=0.62)$.

Cells positive for EG2 were very scarce in all TBB analysed and no statistically significant differences were found between the groups (data not shown).

Inflammatory cells and activation markers in bronchoalveolar lavage fluid

BAL differential cell counts showed a significantly increased percentage of neutrophils in patients with BOS (median $12.0 \%$, range 1-96.5\%) compared with controls (median $3.75 \%$, range $0.5-7 \%, \mathrm{p}<0.002$ ). This difference was still valid when the subject with BOS $3 \mathrm{~B}$ and $H$. influenzae infection in BAL was excluded $(\mathrm{p}=0.003)$. Percentages of eosinophils, lymphocytes and macrophages were not statistically different between the groups (table 2).

BAL fluid levels of MPO were significantly increased in patients with BOS (median $528 \mu \mathrm{g} \cdot \mathrm{L}^{-1}$, range 9-11,000 $\mu \mathrm{g} \cdot \mathrm{L}^{-1}$ ) compared with controls (median $19 \mu \mathrm{g} \cdot \mathrm{L}^{-1}$, range 2-328 $\mu \mathrm{g} \cdot \mathrm{L}^{-1}, \mathrm{p}<0.001$ ) (fig. 3). When the subject with $H$. influenzae infection was excluded, the statistical difference was still valid $(\mathrm{p}<0.001)$. BAL levels of ECP were also higher in patients with BOS (median $40 \mu \mathrm{g} \cdot \mathrm{L}^{-1}$, range 0-200 $\mu \mathrm{g} \cdot \mathrm{L}^{-1}$ ) than in controls (median $2 \mu \mathrm{g} \cdot \mathrm{L}^{-1}$, range 0 $47 \mu \mathrm{g} \cdot \mathrm{L}^{-1}, \mathrm{p}=0.003$ ) (fig. 3).

BAL fluid levels of IL-8 were significantly increased in patients with BOS (median $936 \mathrm{ng} \cdot \mathrm{L}^{-1}$, range 30-4,040 ng. $\mathrm{L}^{-1}$ ) compared with controls (median $10 \mathrm{ng} \cdot \mathrm{L}^{-1}$, range 10 $1,040 \mathrm{ng} \cdot \mathrm{L}^{-1}, \mathrm{p}<0.001$ ) (fig. 3).

BAL levels of albumin did not differ statistically between the groups (BOS, median $51 \mu \mathrm{g} \cdot \mathrm{L}^{-1}$, range 25-320 $\mu \mathrm{g}$. $\mathrm{L}^{-1}$, controls, median $77 \mu \mathrm{g} \cdot \mathrm{L}^{-1}$, range $\left.40-248 \mu \mathrm{g} \cdot \mathrm{L}-1, \mathrm{p}=\mathrm{Ns}\right)$ (fig. 3).

\section{Antioxidants in BAL fluid}

The percentage differences (between the medians) of BAL fluid concentrations of ascorbate were $45 \%$, urate $13 \%$ and GSH $41 \%$ lower in BOS patients than in those without BOS (table 3). In contrast, BAL fluid GSSG was $860 \%$ higher in BOS patients.

Table 2. - Bronchoalveolar lavage fluid differential cell count in lung transplant recipients with and without bronchiolitis obliterans syndrome (BOS)

\begin{tabular}{lcccc}
\hline $\begin{array}{l}\text { Differential cell } \\
\text { count }\end{array}$ & $\begin{array}{c}\text { Neutrophils } \\
\%\end{array}$ & $\begin{array}{c}\text { Eosinophils } \\
\%\end{array}$ & $\begin{array}{c}\text { Lymphocytes } \\
\%\end{array}$ & $\begin{array}{c}\text { Macrophages } \\
\%\end{array}$ \\
\hline With BOS & $12.0(1-96.5)^{+}$ & $0.0(0-4)$ & $5.5(2.5-5.0)$ & $62.5(1-95)$ \\
Without BOS & $3.75(0.5-7)$ & $0.0(0-1)$ & $22.5(0.5-42)$ & $74.0(55-96)$ \\
\hline
\end{tabular}

Values are shown as median (range). ${ }^{*} \mathrm{p}<0.002$, significant difference from patients without BOS. 

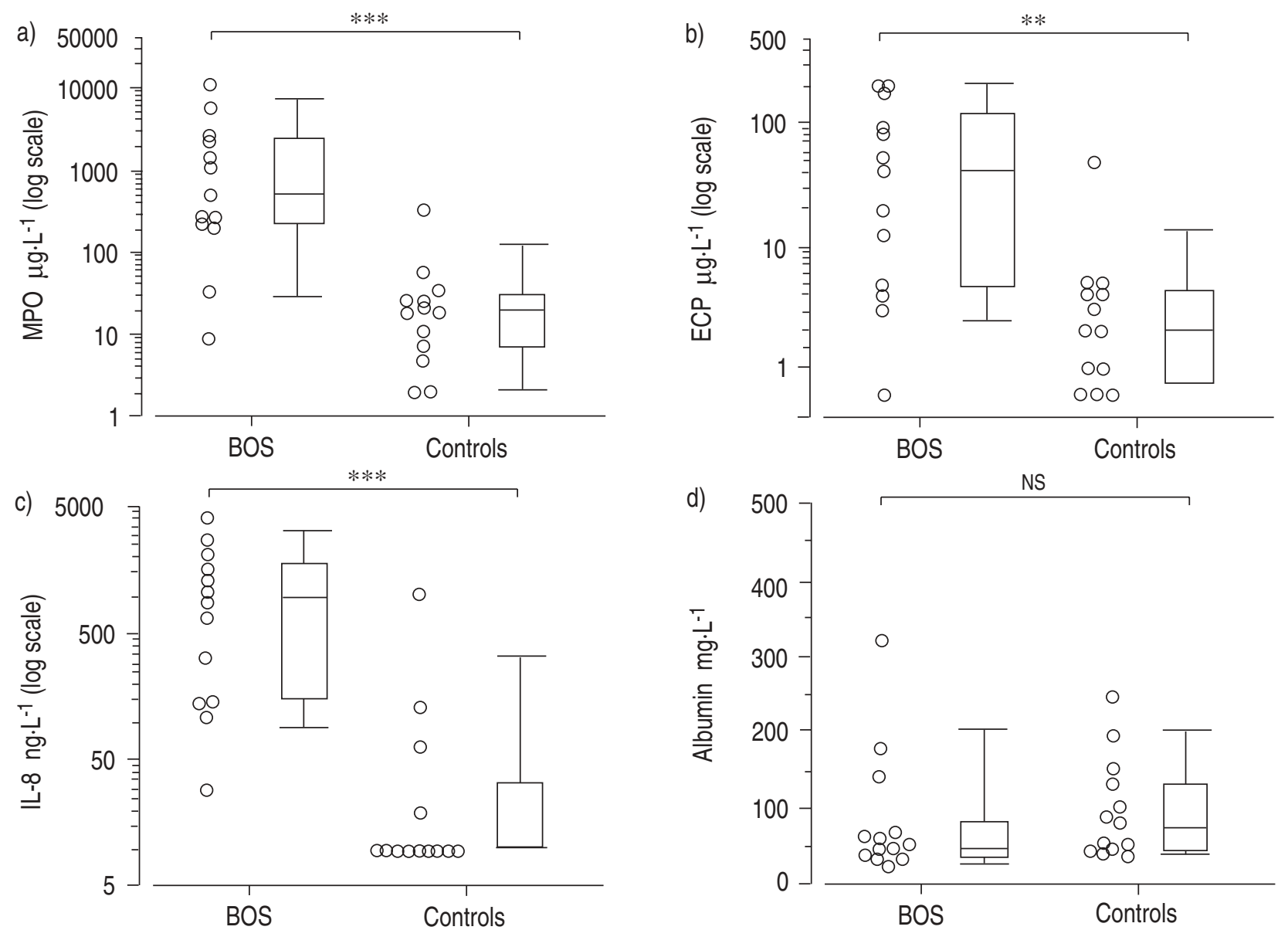

Fig. 3. - Bronchoalveolar lavage (BAL) fluid levels of: a) myeloperoxidase (MPO); b) eosinophil cationic protein (ECP); c) interleukin-8 (IL-8); and d) albumin in patients with bronchiolitis obliterans syndrome (BOS) and controls without BOS. Data are presented as single values and box plots displaying the median value (50th percentile), the corresponding 10th, 25th, 75th and 90th percentiles on either side of the median and the outlying values of the analysed variables. $* *: \mathrm{p}<0.01 ; * * *: \mathrm{p}<0.001$; Ns: nonsignificant difference between groups.

Table 3. - Bronchoalveolar lavage fluid antioxidant levels in lung transplant recipients with and without bronchiolitis obliterans syndrome (BOS)

\begin{tabular}{lcccc}
\hline & $\begin{array}{c}\text { Ascorbic acid } \\
\mu \mathrm{mol} \cdot \mathrm{L}^{-1}\end{array}$ & $\begin{array}{c}\text { Uric acid } \\
\mu \mathrm{mol} \cdot \mathrm{L}^{-1}\end{array}$ & $\begin{array}{c}\text { Reduced glutathione }(\mathrm{GSH}) \\
\mu \mathrm{mol} \cdot \mathrm{L}^{-1}\end{array}$ & $\begin{array}{c}\text { Oxidized glutathione (GSSG) } \\
\mu \mathrm{mol} \cdot \mathrm{L}^{-1}\end{array}$ \\
\hline With BOS & $0.31(0-1.45)$ & $2.64(1.3-8.4)$ & $1.17(0.36-5.47)$ & $0.096(0.01-1.30)$ \\
Without BOS & $0.56(0-4.12)$ & $3.04(1.4-13.6)$ & $1.97(0.59-4.97)$ & $0.01 \quad(0.01-0.440)$ \\
\hline
\end{tabular}

Values are shown as median (range).

\section{Correlations}

BAL fluid levels of IL-8 correlated significantly with percentages of neutrophils $(\mathrm{p}<0.01, \mathrm{r}=0.58)$, levels of MPO $(\mathrm{p}<0.001, \mathrm{r}=0.91)$ and GSSG concentration $(\mathrm{p}<0.05, \mathrm{r}=$ $0.45)$. IL-8 correlated significantly with levels of ECP ( $p<$ $0.001, r=0.81$ ), but not with percentages of eosinophils (NS, $\mathrm{r}=0.21$ ). Percentages of neutrophils in BAL fluid correlated positively with MPO $(\mathrm{p}<0.05, \mathrm{r}=0.61)$ and negatively with GSH concentration $(\mathrm{p}<0.05, \mathrm{r}=-0.37)$.

\section{Discussion}

In the present study convincing evidence was found of neutrophil granulocyte activation both in BAL fluid and in transbronchial biopsies obtained from lung transplanted subjects with a diagnosis of BOS. Increased BAL fluid levels of MPO, a neutrophil oxidative enzyme, as well as low levels of the airway antioxidants ascorbic acid, urate and reduced glutathione indicated increased oxidative stress in BOS patients.

$\mathrm{OB}$ in BOS is characterized histopathologically by chronic inflammatory changes both in the bronchiolar wall and intraluminally with fibrotic granulation tissue [3]. The inflammatory process involves a variety of cells, but so far most studies have focused on the lymphocyte. Some investigators have observed increased percentages of neutrophils in patients with BOS [17, 18], but until recently no detailed analysis of their possible involvement in this disease process has been made. DiGiovine et al. [8] reported both increased numbers of BAL neutrophils and increased BAL levels of IL-8 in patients with BOS and, in 
a smaller number of patients, an immunolocalization of IL-8 to the peribronchial areas of these subjects. The investigators showed neutrophil granulocytes to be associated with BOS and confirmed by neutrophil chemotactic studies that IL-8 accounted for the neutrophil presence in these patients.

The present study extended the findings of DiGiovine $e t$ al. [8]. To control for confounding clinical factors, an attempt was made to match as closely as possible for age, sex, type of lung transplantation, time postsurgery for control bronchoscopy and absence of acute rejection or infection. Using this approach, increased levels of MPO were found in BAL fluid and an increased number of activated neutrophils was found in the bronchial wall of TBB specimens in patients with BOS. Furthermore, strong correlations for both BAL levels of MPO and percentages of neutrophils with degree of monoclonal staining for MPO in TBB specimens were seen. These results support the earlier reported findings of an ongoing activation of the neutrophil granulocytes in patients with BOS.

Signs of eosinophil activation were also present with high levels of ECP in BAL fluid from BOS patients, but the numbers of eosinophils in TBB assessed by staining for EG2 were not increased and did not correlate with BAL ECP levels. High BAL levels of IL-8 were apparent in the subjects with BOS, which is consistent with the knowledge that IL- 8 both attracts and activates neutrophil granulocytes [19]. In accordance with earlier studies [20, 21], correlation analysis revealed strong associations between IL-8 levels and both MPO and ECP levels in BAL fluid, suggesting a primary role for this chemotactic factor in granulocyte inflammatory responses in BOS. However, it should be pointed out that it is not known whether neutrophil activation contributes directly to the pathogenesis of BOS or is merely an epiphenomenon or a sign of late inflammatory cell influx after the disease is already established.

Owing to the increased numbers of activated granulocytes in the lungs of BOS patients it was hypothesized that they would be generating large amounts of reactive oxygen species which, in turn, would lead to depletion of resident lung antioxidant defences. Hypochlorous acid ( $\mathrm{HOCl})$ generated by MPO is able to oxidize thiol groups in molecules such as glutathione and extracellular ascorbic acid can be oxidatively depleted by neutrophil-derived reactive oxygen species [22]. No significant differences in the concentrations of any of the BAL fluid antioxidants examined were found between patients with BOS and those without, but this is probably due to considerable interindividual variability in the measures (which is not due to variable BAL fluid return) and the relatively small study size. However, concentrations of the water-soluble antioxidants (ascorbate, urate and GSH) were generally lower in BOS patients, and GSSG (a marker of oxidative stress) was higher. Moreover, BAL neutrophil percentage correlated positively with MPO and negatively with GSH concentration, indicating that the infiltrating neutrophils were activated and that their presence was associated with depletion of GSH. Importantly, the decreased levels of ascorbate, urate and GSH found in this study were in addition to the already reported low antioxidant status of lung transplant patients $[6,7]$ and, for ascorbic acid, this means that the levels are in the scorbutic range.
To conclude, these findings indicate that granulocyte infiltration and activation, as well as oxidative stress, may play a role in the development and progression of bronchiolitis obliterans syndrome. The results of the study are strengthened by the attempt to control for possible confounding clinical factors. The potential use of myeloperoxidase and interleukin- 8 as markers for disease activity in patients with bronchiolitis obliterans syndrome needs to be evaluated by longitudinal prospective studies. In addition, the finding of low antioxidant levels provides a reasonable basis for further examination of this relationship in a larger cohort of patients, since supplementation therapy could be important.

\section{References}

1. Bando K, Paradis I, Similo S, et al. Obliterative bronchiolitis after lung and heart-lung transplantation. J Thoracic Cardiovasc Surg 1995; 110: 4-14.

2. Yousem SA, Berry G, Brunt E, et al. A working formulation for the standardization of nomenclature in the diagnosis of heart and lung rejection: lung rejection study group. J Heart Transplant 1990; 9: 593-601.

3. Cooper JD, Billingham M, Egan T, et al. A working formulation for the standardization of nomenclature and for clinical staging of chronic dysfunction in lung allografts. J Heart Lung Transplant 1993; 12: 713-716.

4. Kramer MR, Stoehr C, Whang JL, et al. The diagnosis of obliterative bronchiolitis after heart-lung and lung transplantation: low yield of transbronchial lung biopsy. $J$ Heart Lung Transplant 1993; 12: 675-681.

5. Riise GC, Kjellström C, Ryd W, et al. Inflammatory cells and activation markers in BAL during acute rejection and infection in lung transplant recipients: a prospective, longitudinal study. Eur Respir J 1997; 10: 1742-1746.

6. Baz MA, Tapson VF, Roggli VL, Trigt PV, Piantadosi CA. Glutathione depletion in epithelial lining fluid of lung allograft patients. Am J Respir Crit Care Med 1996; 153: 742-746.

7. Williams A, Riise GC, Andersson B, Kjellström C, Schersten H, Kelly F. Compromised antioxidant status of lung transplant recipients. Eur Respir J (submitted).

8. DiGiovine B, Lynch JPR, Martinez FJ, et al. Bronchoalveolar lavage neutrophilia is associated with obliterative bronchiolitis after lung transplantation: role of IL-8. $J$ Immunol 1996; 157: 4194-4202.

9. Riise GC, Schersten H, Nilsson FN, Ryd W, Andersson B. Activation of eosinophils and fibroblasts assessed by eosinophil cationic protein and hyaluronan in BAL. Association with acute rejection in lung transplant recipients. Chest 1996; 110: 89-96.

10. Ljungman P, Griffiths P. Definitions of cytomegalovirus infection and disease. In: Michelson S, Plotkin S, eds. Multidisciplinary Approach to Understanding Cytomegalovirus Disease. Amsterdam, Excerpta Medica, 1993; pp. 233-237.

11. Hsu S, Raine L, Fanger H. Use of avidin-biotin peroxidase complex $(\mathrm{ABC})$ in immunoperoxidase techniques. A comparison between $\mathrm{ABC}$ and unlabelled antibody (PAP). J Histochem Cytochem 1981; 29: 577-580.

12. Peterson CGB, Jömvall H, Venge P. Purification and characterization of eosinophil cationic protein from normal human eosinophils. Eur J Haematol 1988; 40: 415- 
423.

13. Venge P, Strömberg A, Braconier JH, Roxin LE, Olsson I. Neutrophil and eosinophil granulocytes in bacterial infection. Sequential studies of cellular and serum levels of granule proteins. Br J Haematol 1978; 38: 475483.

14. Iriyama K, Yoshiura M, Iwamoto T, Ozaki Y. Simultaneous determination of uric acid and ascorbic acids in human serum by reversed-phase high-performance liquid chromatography with electrochemical detection. Anal Biochem 1984; 141: 238-243.

15. Kelly FJ, Blomberg A, Frew AJ, Holgate ST, Sandstrom T. Antioxidant kinetics in lung lining fluid following exposure of humans to nitrogen dioxide. Am J Respir Crit Care Med 1996; 154: 1700-1705.

16. Tietze F. Enzymic method for quantitative determination of nanogram amounts of total and oxidized glutathione. Anal Biochem 1969; 27: 502-522.

17. Griffith BP, Paradis IL, Zeevi A, et al. Immunologically mediated disease of the airways after pulmonary transplantation. Ann Surg 1988; 208: 371-378.

18. Maurer JR, Gouch E, Chamberlain DW, Patterson GA, Grossman RF. Sequential bronchoalveolar lavage studies from patients undergoing double lung and heart-lung transplant. Transplant Proc 1989; 21: 2585-2587.

19. Baggiolini M, Walz A, Kunkel SL. Neutrophil-activating peptide-1/interleukin-8, a novel cytokine that activates neutrophils. J Clin Invest 1989; 84: 1045-1049.

20. Shute J. Interleukin-8 is a potent eosinophil chemo-attractant. Clin Exp Allergy 1994; 24: 203-206.

21. Riise GC, Ahlstedt S, Larsson S, et al. Bronchial inflammation in chronic bronchitis assessed by measurement of cell products in bronchial lavage fluid. Thorax 1995; 50: 360-365.

22. Stocker R, Frei B. Endogenous antioxidant defences in human blood plasma. New York, Academic Press, 1991; pp. 213-243. 